\title{
Schwannoma of the Common Peroneal Nerve - A Rare Incidence
}

\author{
Tan JA, Levin KB, Rhani SA ( $₫)$, Hisam A \\ Department of Orthopaedics and Traumatology, Faculty of Medicine, Universiti Kebangsaan Malaysia \\ Medical Centre, Jalan Yaacob Latif, Bandar Tun Razak, 56000 Cheras, Kuala Lumpur, Malaysia.
}

\begin{abstract}
Schwannomas are benign tumours arising from neurilemmal cells which forms the myelin sheath of peripheral nerves. It usually occurs in the head, but may be found in the brachial plexus and sciatic nerve. Common peroneal nerve schwannoma are rare. We report a case of a middle age gentleman who presented with pain and swelling over the right popliteal fossa with associated right radicular pain of the anterolateral leg and weakness of ankle dorsiflexion. Examination revealed a $3 \times 2 \mathrm{~cm}$ lump behind the posterolateral aspect of the right knee with positiveTinel's sign upon tapping of the lump, sensory deficit over the anterolateral aspect of the leg and the ankle dorsiflexors had a muscle power of grade 3. Magnetic resonance imaging (MRI) of the right leg revealed a well circumscribed, oval lesion located along the pathway of the common peroneal nerve homogenously hypointense on T1-weighted images and heterogeneously hyperintense on T2 weighted images compared to the muscles. The lesion was not surpressed on fat suppression sequences. Intra-operatively, we noted that the schwannoma was in continuity with the common peroneal nerve. The patient underwent excision of the schwannoma. Post operatively, the pain reduced remarkably but patient suffers from numbness and right foot drop as a complication of the tumour. This case highlights the rarity of common peroneal nerve schwannoma which presents with neuropathic symptoms complicated with right foot drop.
\end{abstract}

Keywords: Common peroneal neuropathy, complication, nerve sheath neoplasms, schwannoma, neurilemmoma

\section{Correspondence:}

Shaharuddin Abd. Rhani. Department of Orthopaedics and Traumatology, Faculty of Medicine, Universiti Kebangsaan Malaysia Medical Centre, Jalan Yaacob Latif, Bandar Tun Razak, 56000 Cheras, Kuala Lumpur, Malaysia. Tel: +603-91457330 Fax: +603-91726473 E-mail: drshahar@ppukm.ukm.edu.my

\section{Introduction}

Schwannomas are slow growing, well capsulated, benign tumours that arise from neurilemmal cells which form the myelin sheath of peripheral nerves. It occurs more in females and usually in the fourth and fifth decade of life. It is the most common solitary nerve tumour in the body with the most common site being in the head but may also be found in the brachial plexus and the sciatic nerve. Rarely, it presents in the lower extremities, but if it does, it usually arises from the deep layers of the foot (1). Unlike Neurofibromas, Schwannomas are benign and have less than one percent chance to become malignant in the form of a neurofibrosarcoma. Patients presenting with multiple
Schwannomas are usually associated with type 2 Neurofibromatosis or Schwannomatosis (2). Traditionally it is believed that Schwannomas do not breech the fascicles of the nerve, instead, it just sits on the myelin sheath. Hence, most patients are asymptomatic until a lump is noted, or when nerve irritation occurs secondary to mechanical compressive effects (3). We report a rare case of a patient presenting with a Schwannoma over the right common peroneal nerve, with the tumour found to be in continuity with the fascicles of the nerve during surgical dissection. The incidence of common peroneal nerve schwannoma is not known, however to date, there are only less than 10 known case reports in the literature since the year $1995(1,3)$. 


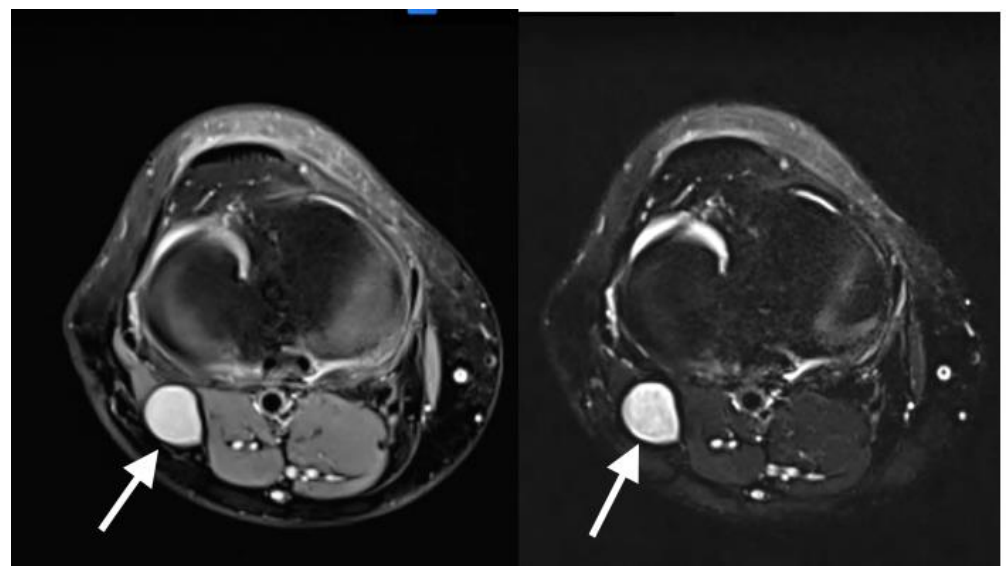

(a)

(b)

Figure 1: a) Sagittal T2-weighted MRI showing a hyperintense lesion along the common peroneal nerve lying between the biceps femoris tendon and lateral head of gastrocnemius muscle (arrow); b) Lesion was not suppressed by fat suppression sequence. (arrow).

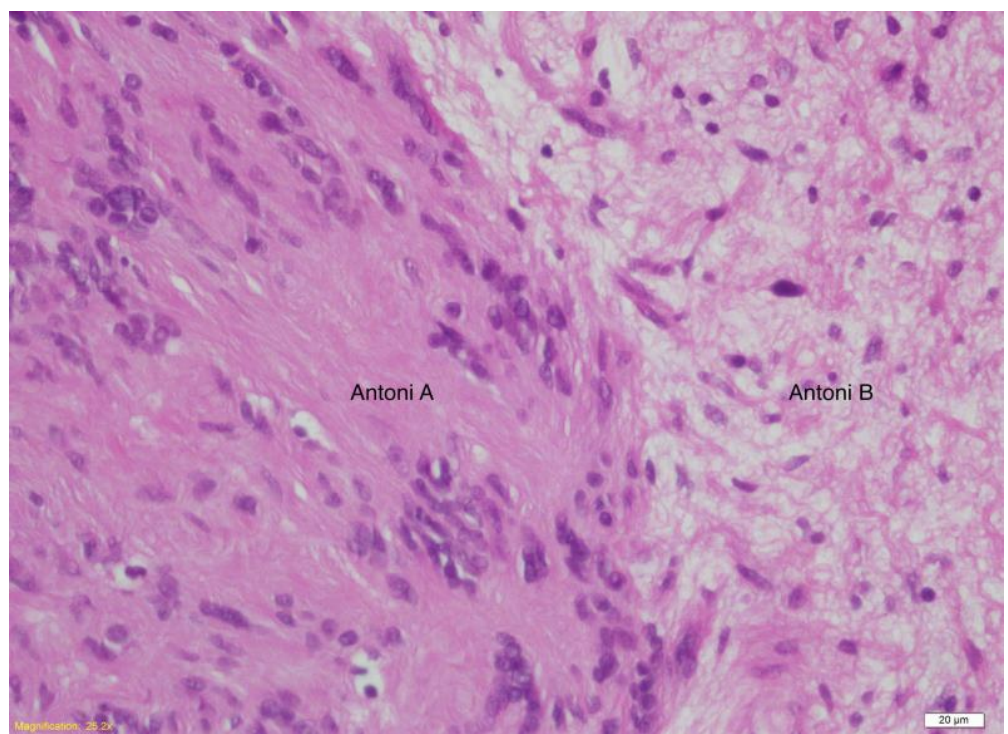

Figure 2: Biphasic tumour composed of compact areas of spindle cells (Antoni A) with palisading Verocay bodies and loosely cellular areas (Antoni B). Maginification of image: 25X

\section{Case Report}

A 50-year-old gentleman was referred to the orthopaedic clinic for pain and swelling over the right popliteal fossa which was gradually increasing in size over the past 6 months. It was associated with worsening radicular pain along the anterolateral aspect of the right leg which was more severe on flexion of the knee. Other than that, he also complained of weakness over the right ankle on dorsiflexion. On examination, he was found to have a $3 \times 2 \mathrm{~cm}$ lump behind the posterolateral aspect of the right knee. The lump was firm in consistency, deep to the fascia and mobile. Tinel's sign was positive upon tapping of the lump, there was sensory deficit over the anterolateral aspect of the leg and the ankle dorsiflexors had a muscle power of grade 3 .
Magnetic resonance imaging (MRI) of the right leg revealed a well circumscribed, oval lesion located along the pathway of the common peroneal nerve. It is homogenously hypointense on T1-weighted images. and heterogeneously hyperintense on T2 weighted images were heterogenously hyperintense compared to the muscles (Fig. 1a). The lesion was not suppressed on fat suppression sequences (Fig. 1b).

In view of the close proximity of the tumour to the common peroneal nerve, the possibility of developing right foot drop after excision had been explained to the patient. Despite that, he was willing to proceed with the surgery as he was more bothered by the neuropathic pain. Patient underwent excision biopsy of the lesion under general anaesthesia. A linear incision was centred over the swelling, exposing the 
tumour which was surrounding the common peroneal nerve. Meticulous dissection of the epineurium was done until the shiny surface of the tumour was identified. During the attempt to remove the tumour as a whole, one of the nerve fascicle was found to be in continuity with it. Multiple attempts to isolate the tumour tissues from the fascicles were not successful, which ended up with damage of the affected fascicle along with removal of the tumour. The tumour had a smooth surface, a distinct capsule and was firm in consistency. Its cross-sectional surface was yellowish in colour. It was subjected to histopathological examination (HPE) which showed a mass composing of two components which are the compact spindle cells in short interlacing fascicles with Verocay bodies (Antoni A) and the less cellular areas with spindle to oval cells which are haphazardly arranged in loose matrix (Antoni B) (Fig. 2). There is no evidence of malignancy and findings correspond to a Schwannoma.

Post-operatively, there was tremendous pain reduction however, the patient developed right foot drop and numbness over the anterolateral aspect of the leg. He was treated with ankle foot orthosis for the foot drop. At 6 months' follow-up, the neuropathic pain did not recur but the motor and sensory deficit persisted, and he refused for further management.

\section{Discussion}

Traditionally, it is believed that Schwannomas do not breech the fascicles of the nerve but instead, it just sits on the myelin sheath. Hence surgical excision rarely aggravates nerve function and most patients remain asymptomatic until a lump is noted, or when nerve irritation occurs secondary to mechanical compressive effects (4). However, in the above case, one of the fascicle from the common peroneal nerve was found to be in continuity with the tumour. More recent studies have shown that larger Schwannomas tend to be in continuity with one or more fascicles of the affected nerve, and hence are at higher risk of nerve damage during excision. Based on this, the authors recommend early excision of the tumour if it is involving a major peripheral nerve (5). Kim et al. carried out a study to assess surgical outcomes of Schwannomas arising from major peripheral nerves in the lower limbs, and reported that 2 out of 30 patients developed persistent motor weakness and marked sensory impairment post excision. It was also noted that surgical enucleation failed in both these patients as the tumour was in continuity with the affected nerve, resulting in permanent nerve damage (6).
Magnetic Resonance Imaging (MRI) is useful to ascertain the location of the tumour in relation to the nerve, so as to enable the surgeon to plan his surgery. Schwannomas usually appears to be isointense on T1weighted sequence and heterogeneously hyperintense on T2-weight sequence in relation to the muscles. It is also remains hyperintense on fat suppress sequences (7). Nerve conduction studies and electromyography may be carried out to assess presence and degree of peripheral nerve dysfunction, as a baseline preoperatively. Despite the wide range of investigatory modalities, there were none useful to predict neurological outcomes post-operatively. The only significant relationship that could be established in regards to post-operatively neurological deficit was the size of the tumour and also those with a longer history (6).

On histopathological examination, Schwannomas exhibit certain distinct characteristics. It usually composes of 2 components which are described as the Antoni A component and the Antoni B component. The Antoni A component has compact spindle cells with collagen matrix arranged into Verocay bodies whereas the Antoni B component presents as spindle or oval shaped cells with haphazard arrangement on a loose mucinous matrix. Features suggestive of malignancy would include high cellular density, high mitotic figures and nuclear atypia (8).

Although Schwannoma is the most common solitary nerve tumour in the body, it may also occur at multiple sites, and if so, is usually associated with Type 2 Neurofibromatosis (NF2) or Schwannomatosis. The diagnostic criteria for NF2 include presence of bilateral vestibular schwannomas, a first degree family member with NF2, and a young patient under the age of 30 presenting with unilateral vestibular Schwannoma or has two or more of the following abnormalities: meningioma, glioma, Schwannoma, juvenile posterior subcapsular lenticular opacities, or juvenile cortical cataract (9). Schwannomatosis is used to describe conditions presenting with multiple Schwannoma in the absence of any stigmata of Neurofibromatosis. It is important to distinguish between these 2, as Neurofibromatosis is more prone to malignant transformation as compared to Schwannomatosis which is almost never malignant.

\section{Conclusion}

In general, the incidence of Schwannoma in the lower limbs are extremely rare with only a few reported cases. It is long believed that surgical resection of 
Schwannomas results in resolution of symptoms and post-operative nerve damage is unusual as the tumour only sits on the nerve without breeching its' fibres. However, more recent studies have suggested otherwise; that excision of larger Schwannomas could result in post-operative neurological damage. Therefore, all patients undergoing excision should be explained regarding this complication and surgeons should pay more attention during dissection of large sized Schwannomas as they are associated with higher risk of fascicular damage. In patients presenting with solitary Schwannoma, a high index of suspicion is required to look for underlying Neurofibromatosis or Schwannomatosis.

\section{References}

1. Mahajan M, Sharma R, Sharma P, Gupta A. Schwannoma of Superficial Peroneal Nerve Presenting as Leg Pain. JCR 2012; 2(2): 79-82.

2. Harbaugh K, Smith P, Towfighi J. Schwannomatosis in a patient with a pelvic mass. Neurosurg Focus 2007; 22(6): E8.

3. Öz TT, Aktaş B, Özkan K, Özturan B, Kilic B, Demiroğlu M. A Case of Schwannoma of the Common Peroneal Nerve in the Knee. Orthop Rev (Pavia) 2017; 9(1): 6825.
4. Joyce M, Laing AJ, Mullet $\mathrm{H}$, et al. Multiple schwannomas of the posterior tibial nerve. Nerve Foot Ankle Surgery 2002; 8(2): 101-3.

5. Park MJ, Seo KN, Kang HJ. Neurological deficit after surgical enucleation of schwannomas of the upper limb. J Bone Joint Surg Br 2009; 91(11):1482-6.

6. Kim SM, Seo SW, Lee JY, Sung KS. Surgical outcome of Schwannomas arising from major peripheral nerves in the lower limb. Int Orthop 2012; 36(8): 1721-5.

7. Hems TE, Burge PD, Wilson DJ. The role of magnetic resonance imaging in the management of peripheral nerve tumours. J Hand Surg $\mathrm{Br}$ 1997; 22(1): 57- 60.

8. Rodriguez FJ, Folpe AL, Giannini C, Perry A. Pathology of peripheral nerve sheath tumors: diagnostic overview and update on selected diagnostic problems. Acta Neuropathol 2012; 123(3): 295-319.

9. Gutmann DH, Aylsworth A, Carey JC, et al. The diagnostic evaluation and multidisciplinary management of neurofibromatosis 1 and neurofibromatosis 2. JAMA 1997; 278(1): 51-7. 